Portland State University

PDXScholar

Engineering and Technology Management

Faculty Publications and Presentations

$10-8-2018$

\title{
Effects of Future Connected Autonomous Vehicles on Freeway Congestion Using Fuzzy Cognitive Mapping
}

Hakan Kutgun

Portland State University

Vivian Du Pont

Portland State University

Henry Janzen

Portland State University

Follow this and additional works at: https://pdxscholar.library.pdx.edu/etm_fac

Part of the Engineering Commons

Let us know how access to this document benefits you.

\section{Citation Details}

H. Kutgun, V. D. Pont and H. Janzen, "Effects of Future Connected Autonomous Vehicles on Freeway Congestion using Fuzzy Cognitive Mapping," 2018 Portland International Conference on Management of Engineering and Technology (PICMET), Honolulu, HI, 2018, pp. 1-7.

This Article is brought to you for free and open access. It has been accepted for inclusion in Engineering and Technology Management Faculty Publications and Presentations by an authorized administrator of PDXScholar. Please contact us if we can make this document more accessible: pdxscholar@pdx.edu. 


\title{
Effects of Future Connected Autonomous Vehicles on Freeway Congestion Using Fuzzy Cognitive Mapping
}

\author{
Hakan Kutgun, Vivian Du Pont, Henry Janzen \\ Engineering and Technology Management Department, Portland State University, Portland, OR, USA
}

\begin{abstract}
Continuing population growth and urbanization are projected to add 2.5 billion people to the world's urban population by 2050 [1]. It is evident that this will increase traffic congestion especially in the urban areas, which will bring economic, safety, environmental and quality of life challenges. There are various organizations looking for possible solutions to reduce the impact of future congestion by long term planning [2], most of these studies don't take into account emergence of disruptive technologies. The concept of vehicles with autonomous driving and online connectivity capabilities, namely, connected autonomous vehicles (CAVs) is an emerging technology [3] which may contribute to the solution of this problem through adoption. This paper aims to shed light on effect of different levels of CAV adoption on congestion through scenario planning with fuzzy cognitive mapping. Different future scenarios on CAV adoption based on research and development being done on CAV technology [3] are run through a fuzzy cognitive model of congestion developed through detailed literature review. Results indicate CAV adoption provides an opportunity for reducing congestion. Therefore suggesting, investing in CAV enabling upgrades of existing roads, and giving incentives for CAV adoption, is a viable option for city planners' and local governments' project portfolios to reduce congestion.
\end{abstract}

\section{INTRODUCTION}

Road congestion continues to grow, exasperating issues such as increased travel time, negative economic impact, increasing the number of accidents, negative environmental impact, and decreasing driver's well being. A 2004 study [4] reveals that in the early 2000's the cost of congestion in the US was $\$ 7.8$ billion annually. Many options are being considered to reduce congestion, such as additional lanes, improved traffic controls, encouraging alternate means of transportation. Recent developments in car technology introducing low levels of autonomous cars with the goal to reach fully autonomous cars, holds promising potential to reduce congestion. Autonomous vehicles can reduce vehicle to vehicle spacing [5] and provide safer vehicle operation reducing accidents [6], both of which have the potential to reduce congestion. Many car manufactures are engaged in autonomous car development [3] achieving significant strides. Currently cars are available with adaptive cruise control and automated lane changing. Fully autonomous cars are projected to be the dominant vehicle configuration by 2040 [7]. This paper examines the potential impact autonomous cars could have on road congestion using Fuzzy Cognitive Mapping.

\section{A. Impact of Road Congestion}

In 2014, the average American spent 42 hours in traffic congestion delays which equates to $\$ 132 \mathrm{~B} /$ year in lost labor and fuel costs. Commercial trucking congestion adds $\$ 28 \mathrm{~B}$ in labor and fuel costs for a total of $\$ 160 \mathrm{~B} /$ year. In 1982 , the yearly auto commuter congestion delay was $18 \mathrm{hrs}$ and the total cost including commercial trucks in 2014 dollars was $\$ 42 \mathrm{~B} /$ year. There has been a steady increase in congestion. With the growing urbanization trend, congestion and associated costs are expected to increase to $\$ 192 \mathrm{~B} / \mathrm{yr}$ by 2020 (in 2014 dollars) [3].

The stop and go nature of congestion traffic reduces engine operating efficiency, increasing car emissions vs the steady flow of cars in non-congested areas. A study carried out in Southern California estimates that congestion reduction measures could reduce vehicle CO2 emissions by 7 - 24\% [8]. This is a wide range, but it shows that through congestion reduction there can be significant reduction in $\mathrm{CO} 2$ emissions.

The number of traffic accidents increases as congestion worsens [9]. This stands to reason as the stop and go nature of congestion introduces more activity on the part of the driver to brake and accelerate, introducing more potential for error. More congestion often causes drivers to change lanes in effort to find a faster lane. Lane maneuvers again increases the potential for driver error. In addition, accidents cause secondary accidents due to flow interruption and 'rubber necking' further increasing the frequency of accidents [9].

Finally a person's well being and productivity decline when the commute regularly battles congestion [10]. There are mixed reports on whether the congestion has significant impact on general wellness and productivity, but there is growing evidence that traffic congestion has a negative impact on well being. [11]

These negative impacts due to road congestion make road congestion a major issue that drive government, industry, academia and general public searching for ways to reduce it.

\section{B. Trends in Autonomous Vehicles}

The race is on to develop autonomous cars. The race not only includes the current dominant car OEMs such as Toyota, GM, Ford, Honda etc, but also Silicon Valley companies such as Apple, Google and Tesla, and start-ups such as NuTonomy [12]. The majority of major vehicle OEMs are heavily engaged in autonomous vehicle development currently offering lower level of autonomous vehicle features such as adaptive cruise control, lane assist and lane changing. Navigant Research 
analysis rated the top 18 players in the autonomous vehicle arena noting that many of these companies are currently testing autonomous vehicles public roads. [13]

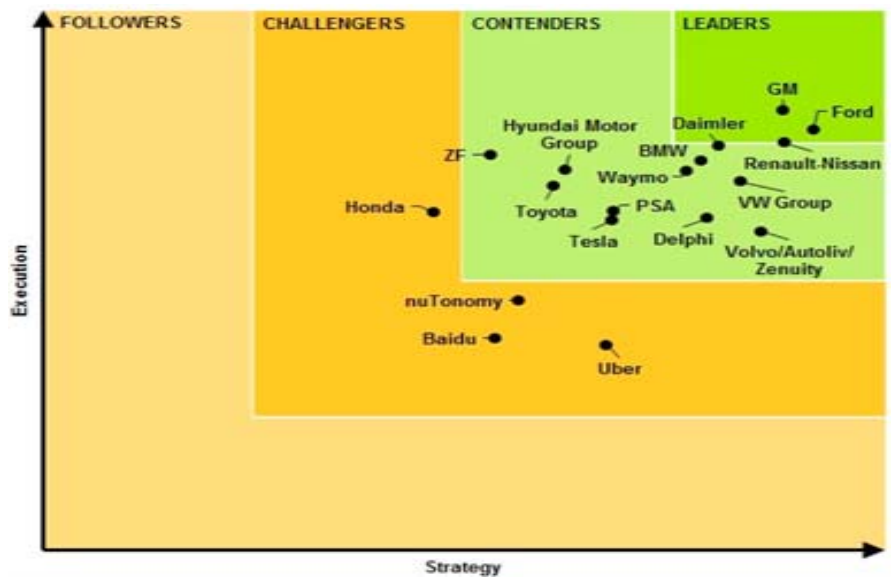

Fig. 1. Industry Engagement in the Autonomous Vehicle Development [3]

Even though the race is on to develop and implement autonomous vehicles, due to safety reasons and the lifecycle of cars, most estimations of autonomous car sales predict it won't be until 2040 before autonomous cars are the dominant seller [7]. See figure 2 for adoption prediction of autonomous vehicles.

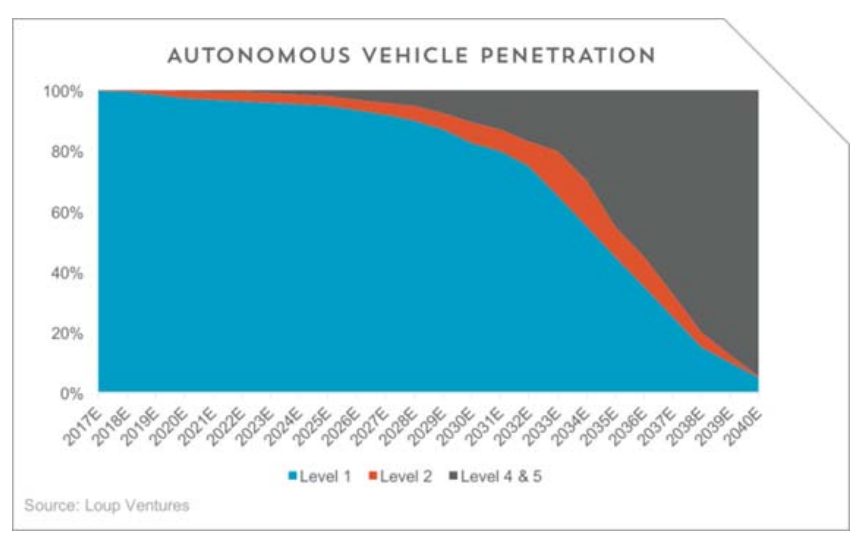

Fig. 2. Adoption Prediction of Autonomous Cars [7]

\section{Scope}

This study focuses on controlled access highways in metropolitan areas, highways connecting cities within 60 miles and traffic corridors. These are the areas where the high density traffic creates significant congestion issues. Secondly this environment was chosen as there is some available research data on autonomous vehicle impact.

Autonomous vehicles considered in this study are level 4 per Society of Automotive Engineering standard 3601. A level 4 autonomous vehicle requires a driver, but the vehicle controls all driving functions, allowing the driver to perform leisure, work or other activities. A level 5 autonomous car does not require a driver, see appendix $\mathrm{A}$ for a description of all autonomous car levels. The large number of factors influencing the impact of even just a level 4 autonomous vehicles on congestion resulted in this study focusing level 4 autonomous vehicles to make this a manageable, and more meaningful study.

\section{METHODOLOGY}

Literature research was carried out to gather information on existing and future autonomous vehicle solutions, and on car congestion studies. After identifying the main causes of congestion as well as collect expert opinion on the subject, knowledge obtained is used to represent causalities in a cognitive map. The authors of this paper conducted literature review in order to provide expert opinion input.

The flowchart of the methodology is given in Figure 3. Causal cognitive maps were chosen as the primary method to develop and explore multiple scenarios related to the adoption of autonomous vehicles. The cognitive mapping technique applied is Fuzzy Cognitive Mapping (FCM), which provides the structure to identify, model and clarify the system behavior. FCM method was introduced by Bart Kosko [14] in 1986, and since then, has gained considerable interest due to their potential to represent structured knowledge and develop model scenarios for complex systems in various fields.

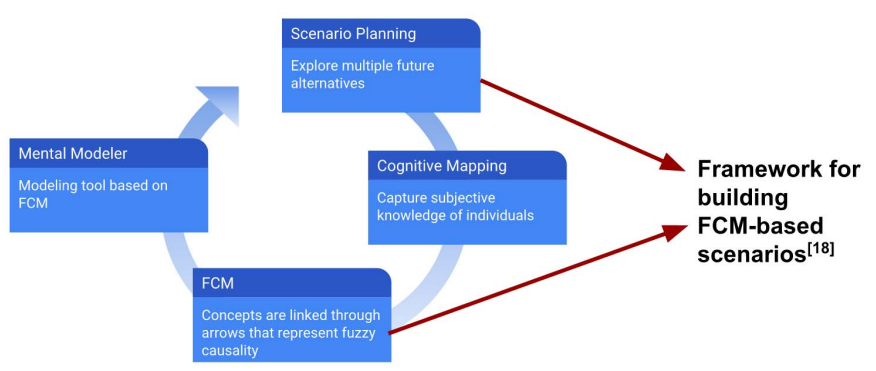

Fig. 3. Flowchart of the Methodology

Once the FCM scenario is developed, a participatory modeling tool for FCM named Mental Modeler [15] was be used to simulate and evaluate the system dynamics in order to determine how the introduction of autonomous vehicles will impact traffic congestion.

\section{A. Fuzzy Cognitive Mapping \& Scenario Planning}

Cognitive maps were first introduced in the 1970s by Robert Axelrod to represent social scientific knowledge [14]. FCM has its origins in concept and cognitive mapping and provides a way to systematically collect and represent knowledge in fuzzy-graph structures.

With FCM, mental models can be created by representing causal relations between elements of a system or environment, and applying fuzzy logic to those causal relationships to express the hazy degrees of causality in the cognitive map. As a result, FCM offers a powerful tool to capture and interpret the complexity and knowledge of an environment or a system as well as understand its conceptual components.

The early identification of future trends and the anticipation of market changes are crucial factors for business to achieve success under uncertainty. In this scenario, the use of techniques to explore multiple future alternatives and address business challenges has become extremely important. 
Scenario planning techniques have raised as one of the most effective approaches to help companies become more flexible and innovative, and make better decisions while dealing with business challenges [16]. Since 1970s, a number of scenario development techniques and methodologies have been proposed and studied [17]. A framework for building FCMbased scenarios proposed by Jetter \& Schweinfort [18], which is comprised of 6 steps integrating two different approaches for Scenario Planning and FCM modeling, has been used as a guideline for this project. For each step, Figure 3 shows the tasks undertaken.

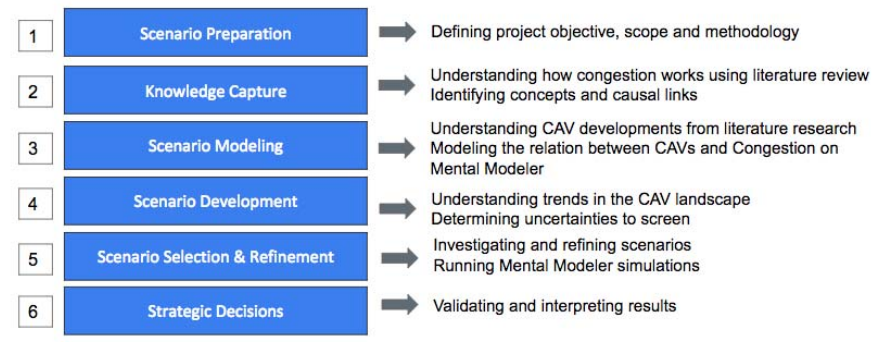

Fig. 4. Figure 4 - Framework for Building FCM-based scenarios

\section{B. Factors Contributing to Congestion}

To shed light on how CAVs might mitigate congestion we need to understand the underlying causes of its occurrence, as a whole. According to a 2003 study by Federal Highway Administration [19], causes of traffic congestion are listed under two categories as recurring and nonrecurring. Recurring causes are given as insufficient capacity, unrestrained demand, ineffective management of capacity whereas non recurring causes are given as incidents, work zones, emergencies, and weather conditions for conventional traffic congestion.

A further study in 2004 [20] depicts the relationship between mobility or congestion (outcome) measures and operations or efficiency (output) measures as given in Figure 5.

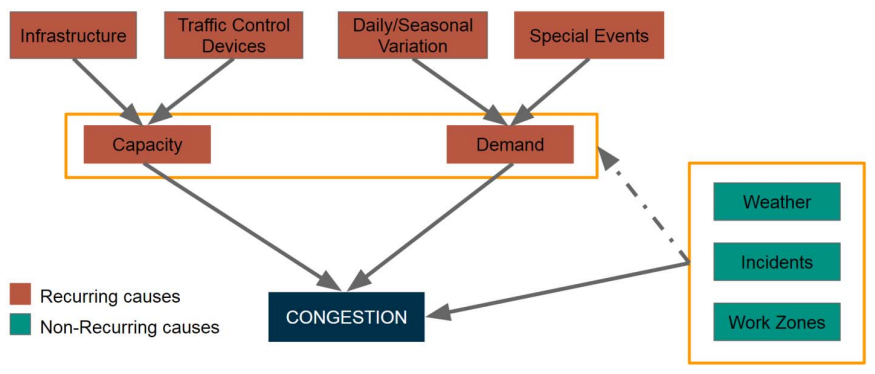

Fig. 5. General Taxonomy of Factors Causing Congestion

We primarily focus on how CAV adoption affects recurring causes of congestion as they are the reason for the chronic congestion.

\section{Recurring Congestion}

These are the factors that cause congestion on an ongoing basis. The continuous nature of these factors is the reason for the chronic congestion on freeways.

\section{1) Capacity}

Since the capacity of freeways are limited at any given time and location, the only way that CAVs have effect on capacity is through improving management of capacity. This includes route and signaling optimization, better decisions made by informed driver (human or machine) and flow pattern of vehicles in traffic [21]. In these areas, 5 factors are identified that CAV technology is expected to improve that would directly affect capacity are: Signal timing optimization, route optimization, efficiency of lane changes, spacing between vehicles and average velocity of vehicles. The connectivity features would especially be useful through signal timing and route optimizations. CAVs can receive the information of any capacity bottlenecks (due to both recurring and non-recurring causes) in advance and road signals can be further optimized by information flowing through the CAVs creating the demand.

\section{2) Demand}

The demand on freeways is primarily driven by number of cars in traffic and how long they stay on the road [21]. This number includes all vehicles in traffic; personal vehicles, freight, car sharing, transit and alike. Transit use directly affects the number of cars as well as demands itself. The time dependent increase in total demand is considered a nondiscriminating factor between different CAV scenarios therefore left out of scope of this study. However, public transit is a different story. Currently only $5 \%$ of US commuters use public transit [22]. This picture changes when considering high density areas. The top four US counties with highest concentration of public transportation are counties in New York, where on average in these counties $57 \%$ of workers use public transportation to travel to work. In Oregon $4.16 \%$ of commuters use public transportation, with $10.7 \%$ commuters in Multnomah county use public transportation [22]. The effect of CAV adoption on car sharing and public transport are taken into account as factors that would change the number of vehicles in traffic through reducing personal vehicle ownership.

\section{Non-recurring Congestion}

These are the factors that cause congestion on the basis of discrete occurrences. The occurrence of these factors is limited to time and space, therefore cause acute congestion on freeways.

\section{1) Incidents}

Primarily caused by driver errors, occurrence of incidents, ie traffic accidents, are also affected by work zones and inclement weather conditions.

\section{2) Inclement Weather}

Although the occurrence of inclement weather is left out of scope of this study, its effects on incidents, driver errors, effective management of capacity and congestion are discriminating factors for future scenarios.

\section{3) Work Zones}

Freeways require regular maintenance and occasional repairs. These activities create work zones which affect capacity, driver errors and congestion. Number of cars in traffic and effective management of capacity affect the consequences of work zones that cause congestion. 


\section{FUTURE SCENARIOS}

Taking current traffic conditions (i.e. almost no CAVs in traffic) as baseline, variations of 2 different future scenarios are considered:

\section{Scenario 1 - High CAV Adoption}

\section{Scenario 2 - Moderate CAV Adoption}

To provide consistency in scenarios identified, trends of T1: Advancing CAV technology [23] and T2: Increasing urbanization [1] are cross matched with 3 future uncertainties: U1: Consumer acceptance of CAVs, U2: Effect of CAVs on car sharing and public transport, U3: Regulations and infrastructure to support CAV use. Although it is evident that $\mathrm{U} 1$ and $\mathrm{U} 2$ will follow direction of $\mathrm{T} 1$ and $\mathrm{T} 2$, no reliable source was found for us to comment on which way U2 will go with CAV adoption. Therefore effect of CAVs on car sharing and public transport are taken into consideration as separate scenarios as shown in Table 1

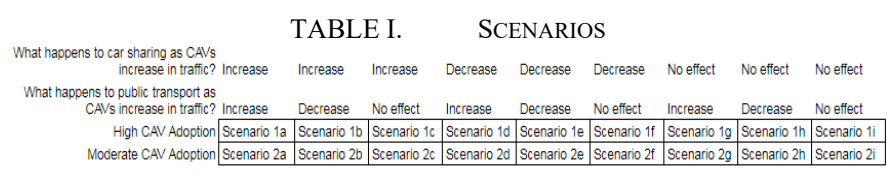

Within each of the primary scenarios, sub-scenarios are run to evaluate the influence of increasing and decreasing use of public transit and car ride sharing. Different scenarios considered are as follows:

Baseline: The world as we live in today. Traffic demand dominated by conventional vehicles running on fossil fuels. Urbanization and congestion are on the rise. Connected drivers exist with a human interface; however smart roadways are limited to interactive signaling, without any direct interface with driving vehicles. Car sharing and electric vehicles are on the rise with a future open to multiple possibilities.

Scenario 1 High CAV Adoption: A world dominated by high adoption of advanced technology. Safe, reliable technical solutions combined mass market with price reductions make electrical drive and CAVs more appealing and widely acceptable. With increasing economic growth and urbanization, demand in a higher quality of life increases rapidly. As most of the vehicles in traffic consist of CAVs, even conventional cars are much more connected. Local and national governments have the vision and resources to respond rapidly to overcome the regulatory challenges as a priority. Transportation budgets are mostly spend on connectivity upgrades of existing roads rather than increasing capacity by traditional means, which with advancements in technology becomes cheaper to do. Roadways are optimized more and more towards CAV use.

Variation 1: Consumers are able and willing to choose tailored transportation choices among an array of on-demand services. Personal car ownership declines.

Variation 2: With increasing economic growth, CAVs become personalized commodities. Cars remain a way of expressing personal identity, even expression of social status with CAVs suited to needs of their owners. Car sharing and public transportation dwindle.
Scenario 2 Moderate CAV Adoption: Although the technical advancements in EV and CAV development continue, they are mostly market driven and commercial use of fully autonomous CAVs is the primary concern. Public have concerns about fully autonomous cars and adoption is limited. Urbanization is almost on par with suburban sprawl making arterial roadways even more critical. There are lanes allocated to fully autonomous commercial cars with smarter infrastructure. People prefer to keep their own cars while car sharing and overhaul are dominated by fully autonomous CAVs. Although connectivity is now mainstream, there aren't as many autonomous cars in traffic as there are conventional cars in personal use. CAVs remain out of reach for most of the public causing a backlash against advanced CAVs as a threat to low-income jobs and given privileges in allocated lanes.

Local and national governments are torn between allocating resources to technological upgrades of existing roadways towards CAV compatibility vs. increasing capacity. Progress regarding regulatory challenge is lagging.

Variation 1: There's steady demand for personal vehicles. Car sharing continues steady without a disruptive spike.

Variation 2: Fed up with challenges of commuting long distances and urbanization, most people prefer to use car sharing and public transport for their commutes.

\section{ANALYSIS}

Through extensive literature review, a model of freeway congestion is constructed as a fuzzy cognitive map (FCM) as seen in Figure 6. The model consists of 15 elements (a.k.a nodes) and 32 connections (a.k.a. vectors).

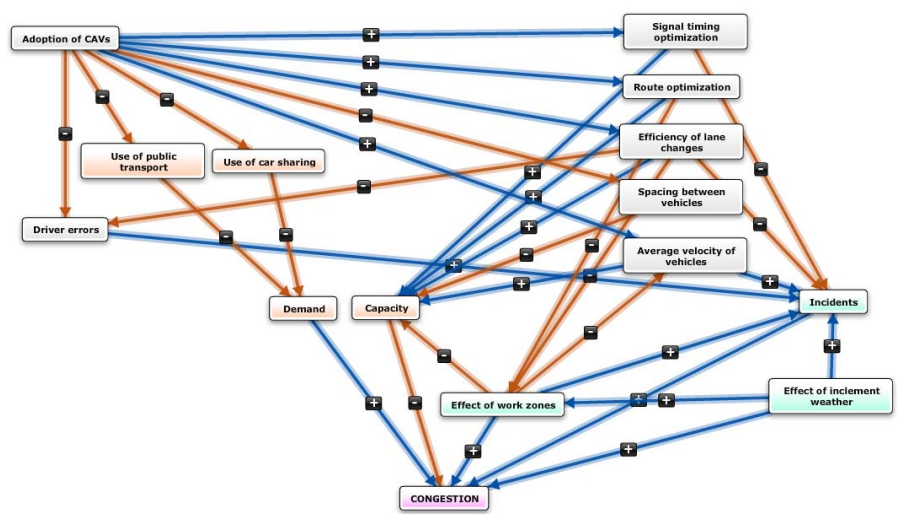

Fig. 6. Freeway Congestion FCM

Although "Effect of inclement weather" has no connection to receive input from "Adoption of CAVs" it is left in the model for the sake of completeness. All vectors connecting the elements have equal strength, differing only as positive or negative. The values for any vector is given as 1 for direct correlation or -1 for a negative correlation between connected elements in the direction of the connecting vector, see appendix $\mathrm{B}$ for all vector inputs.

The only node excited (given initial input) for different scenarios is the "Adoption of CAVs" at the top left corner of the model. The model uses a sigmoid squashing function. A 
few logical check runs conducted on the model identified an input of 0.5 to be the "neutral point" for the input node (Adoption of CAVs). This means that 0.5 value as the input will be considered as the baseline for representing the current state as no CAV adoption. Keeping the maximum value of the input same as the strength of connecting vectors, 1.0 is considered as the representation for Scenario 1 and 0.7 is considered as the representation for Scenario 2.

For the variation of scenarios (a through $\mathrm{i}$ for Scenario 1 and Scenario 2) the value of vectors connecting "Adoption of CAVs" to "Use of public transport" and "Adoption of CAVs" to "Use of car sharing" are rotated between 1, 0 and -1 for all possible combinations. The analysis yielded 18 different values for "Congestion" as shown in Table 2.

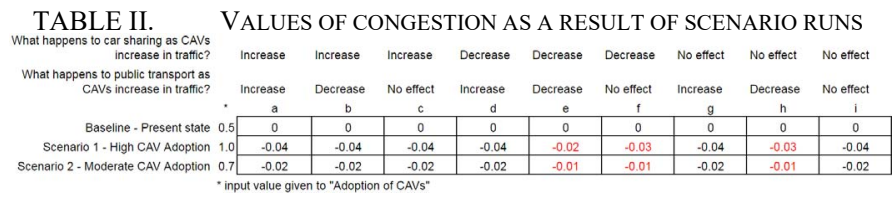

As negative values mean decrease in congestion, results indicate that even moderate $\mathrm{CAV}$ adoption reduces congestion, but not as much as a high adoption scenario. Comparing results on column $\mathrm{i}$ to the rest on Table 2 , it can be concluded that a negative correlation between CAV adoption and public transport \& car sharing is influential, however a positive correlation is not. As either or both of public transport and car sharing decrease, the reduction in congestion also decreases. In some cases (Scenario 2e), decrease in public transport and car sharing as a result of CAV adoption evens out the congestion reduction effect of CAV adoption (Scenarios 2a, 2b, 2c, 2d, 2g, 2i).

\section{Discussion}

The initial mental modeler scenario was run to test the case of increasing the CAV adoption, while not changing other components. To simplify the analysis, the model was constructed with moderate number of components. Components included were thought to be the more significant factors in the CAV and congestion relationship. For example, one component that was not included was the potential increase in miles travelled if CAV usage is increased. Some predict that if CAV's are used, and people can do work, relax or perform other leisure activities while driving. In turn this may result in people living further from business centers and increasing the commute distance. This would effectively add more cars travelling on a road, increasing demand and as a result increase congestion. There are many more examples of factors that could contribute to CAV adoption and congestion, but to not overly burden the initial analysis with undue uncertainty, the model was constructed with a moderate number that were considered dominant components and elements, to give some direction of CAV impact on congestion. With a moderate number of components in the model, the simple case of increasing CAV adoption reflected that congestion was reduced. Since simple binary (either +1 or -1 ) relationship between components was used, the number of components related to a phenomenon influenced the results. For example, in the mental modeler model, the adoption of CAV's are linked to a number of components such as reducing vehicle following distances, more efficient lane changes, higher vehicle speeds, and route optimization. All these components reflect a plausible impact on congestion with CAV adoption, but when using binary component relationships, each of these components add weight to the increase of CAV's. The reason for including the multiple components linked to $\mathrm{CAV}$ adoption is important to communicate all the factors the CAV's impact.

When the mental modeler was run activating only the adoption of CAV's at 0.7 and 1.0, both inputs resulted in a reduction in congestion to -0.02 and -0.04 respectively, with a higher negative congestion value representing a higher reduction in congestion. Using mental modeler, the degree to which the increase in CAV's impacts congestion cannot be determined, but the model output indicates that continued increase in CAV's adoption resulted in a continued reduction in congestion, which aligns with what would be expected.

\section{A. Limitations and Risks}

As with any simulation, the input data is key. Nice colorful outputs can sometime lull users into thinking the results are plausible, when in fact reality is far from what is being modeled. Users and interpreters of mental model results need to keep the validity of input data in mind.

A means to model varying component relationships in different operating conditions is a limitation. Perhaps manually identifying these circumstances and applying different weightings accordingly is a means to get around step shifts in operating conditions.

\section{IMPLICATIONS}

In order to see how the mental modeler responds to a more complex set of components activated in a scenario, different levels of public transportation and car sharing are activated. In addition to activating these components, the relationship of these components was also reversed with respect to CAV adoption from an increase to a decrease relationship. The flipping of the relationship from increasing to decreasing is a realistic reflection of CAV impact. The increased vehicle cost due to CAV's may drive people to use more economical public transportation. Or perhaps the added convenience of automated cars may make CAV cars more attractive and move people away from public transportation and into personal CAV's. Executing the mental modeler scenarios by varying the relationship of public transport and car sharing to decrease/increase/no effect, resulted in mostly predictable outcomes. When either public transportation or car sharing was set as decreased, or set to no effect, congestion worsened. This is logical as public transportation and car sharing is more efficient than a personal vehicle with respect to congestion, thereby reducing the use of either should increase congestion. What was not expected was that if both public transportation and car sharing increase as a result of CAV adoption increase, the expectation is that congestion would be further reduced from the scenario where the adoption of CAV's has no impact on the use of public transportation or car sharing. The mental modeler output result in this scenario did not have an impact on congestion. A possible explanation for this is that the congestion values do not have much spread from one scenario 
to the next and these are rounding errors, or similarly the impact is negligible.

\section{CONCLUSION}

FCM using Mental Modeler provided a relatively simple tool to visualize and predict impact of factors on complex future developments. Following are the CAV and congestion conclusions based on the FCM results:

\section{CAV adoption has the potential to reduce road congestion.}

Even moderate adoption of CAV's reduces road congestion, with higher adoption rates further increasing the reduction of road congestion.

CAV's might offer an effective means to reduce road congestion versus traditional methods of building new roads, expanding existing ones. Cost efficiency of it warrants further study.

\section{FUTURE RESEARCH}

Through experimentation on the model, the binary model (having only 1 and -1 as vector values) is found to be dependent on the number of nodes converging on demand and capacity. Assigning fuzzy values to vectors may address this problem. This would require data to support the weightings. Some data is available, for example there is spacing data comparing a conventional car to an autonomous car, but there would be many component relationships that do not have data. Another tool might be expert input into the model to assign weights to connections between elements. Another method to improve the FCM would be to add more elements, again preferably through expert input. By adding more elements and assigning fuzzy values for interdependencies, the methodology can be expanded to discriminate between different levels of autonomous vehicles. More scenarios can be therefore added to provide a richer variety of possibilities. This would lead to an even wider perspective to understand the effects of CAV adoption. This may pave the way to a more comprehensive study to shed light on effect of CAV adoption on more than just congestion. Also for congestion, at a certain demand, the capacity starts reducing, moving the relationships of the components into a totally different relationship. In the over capacity environment, the benefits of CAV's is diminished and other factors such as mass transit that directly reduce demand play a more significant role. It is not clear how these threshold points could be reflected using FCM.

A case study for verification of the model may be conducted by adoption of this method into a selected country case where traffic jam problem is very critical.

This analysis deserves a more detailed revisit to compare cost of increasing capacity by traditional means (e.g. building new roads, expanding existing ones etc.) vs. technological upgrades to increase CAV adoption and effect of CAV adoption on congestion. Hierarchical decision making models such as HDM [24], AHP [25], and FAHP can help prioritizing the multi-perspective problems in this area (as projects to be selected to be solved) or facilitate the decision making and alternative selection among different solutions.

\section{REFERENCES}

[1] United Nations 2014"World Urbanization Prospects" Department of Economic and Social Affairs, https://esa.un.org/unpd/wup/Publications/Files/WUP2014-Highlights.pdf

[2] Department of Economic and Social Affairs, December 2, 2017

[3] "Traffic Congestion and Reliability, Trends and Advanced Strategies for Congestion Mitigation", Cambridge Systematics, Inc. with Texas Transportation Institute, 2005.

[4] Navigant Research Leaderboard Report "Assessment of Strategy and Execution for 18 Companies", Internet: Retrieved from https://www.navigantresearch.com/research/navigant-researchleaderboard-report-automated-driving December 3, 2017

[5] Schrank., D., Eisele., B., Lomax., T., \& Bak., J. (2015, August 5). “ Urban Mobility Scorecard". Texas A\&M Transportation Institute, v39 Internet: Retrieved from https://doi.org/DTRT06-G0044 December 2, 2017.

[6] US Department of Transportation. 2007, April. Number: FHWA-HRT07-003 Issue No: Vol. 70 No. 5 "Understanding Freight Bottlenecks", Internet: Retrieved from https://www.fhwa.dot.gov/publications/publicroads/07mar/05.cfm December 2, 2017

[7] National Highway Transportation \& Safety Administration . "Automated Vehicles for Safety" , Internet: Retrieved from https://www.nhtsa.gov/technology-innovation/automated-vehicles-safety December 2, 2017

[8] Gene Munster, 2017, September 6 "Auto Outlook 2040: The Rise of Fully Autonomous Vehicles" Loupventures, Internet:. Retrieved from http://loupventures.com/auto-outlook-2040-the-rise-of-fullyautonomous-vehicles/ December 3, 2017

[9] Barth, M., \& Boriboonsomsin, K. (2008, March 3). Real-world CO2 impacts of traffic congestion. Transportation Research Record: Journal of the Transportation Research Board, 2058(1), pgs 163-171.

[10] Kalasova A., \& Stacho, M. (2006, January 4) "Smooth traffic flow as one of the most important factors for safety increase in road transport" Transport, $21(1)$, https://doi.org/10.1080/16484142.2006.9638037

[11] Gino F.,Staats B., Jachimowicz J.,Lee J.,Menges J., 2017. Reclaim Your Life. Harvard Business Review, May-June: pgs 149-153

[12] Hilbrecht, M., Smale, B., \& Mock, S. E. (2014). "Highway to health Commute time and well-being among Canadian adults". World Leisure Journal, 56(2), 151-163. https://doi.org/10.1080/16078055.2014.903723

[13] Litman, T. (2014). Autonomous Vehicle Implementation Predictions: Implications for Transport Planning. Transportation Research Board Annual Meeting, 42(2014), 36-42. https://doi.org/10.1613/jair.301

[14] Muoio, D. 2017, September 27." The 18 companies most likely to get self-driving cars on the road first." Internet Retrieved from http://www.businessinsider.com/the-companies-most-likely-to-getdriverless-cars-on-the-road-first-2017-4/\#17-nutonomy-2 , December 2, 2017

[15] Kosko, B. (1986). "Fuzzy Cognitive Maps". International Journal of Man-Machine Studies. 24: 65-75.

[16] Gray, Steven A.; Gray, Stefan; Cox, Linda J.; Henly-Shepard, Sarah (2013). "Mental Modeler: A Fuzzy-Logic Cognitive Mapping Modeling Tool for Adaptive Environmental Management". 2013 46th Hawaii International Conference on System Sciences. pp. 965-978

[17] Amer, M.; Daim, T.; Jetter, A. (2013). "A review of scenario planning". Futures 46, 23-40

[18] R. Bradfield, G. Wright, G. Burt, G. Cairns, K. Van Der Heijden, (2005). The origins and evolution of scenario techniques in long range business 795-812.

[19] Jetter, A., Schweinfort, W., Building scenarios with Fuzzy Cognitive Maps: An exploratory study of solar energy, 2011 Futures 43 (1), pp. 5266.

[20] Jeffrey F. Paniati. "Using Intelligent Transportation Systems (ITS) to Better Manage Traffic Congestion" Traffic Congestion: Issues and Options Symposium. Internet: https://ops.fhwa.dot.gov/congsymp/sld003.htm, Jun. 26, 2003 [Nov. 1, 2017] 
[21] Cambridge Systematics Inc. with Texas Transportation Institute. "Final Report Traffic Congestion and Reliability: Linking Solutions to Problems",

Internet:

https://ops.fhwa.dot.gov/publications/publications.htm, Jul. 2004 [Nov. $1,2017]$

[22] Cambridge Systematics Inc. with Texas Transportation Institute. "Final Report Traffic Congestion and Reliability: Trends and Advanced Strategies for Congestion Mitigation"

[23] Chase, 2017, September 10. "What Percentage of Americans Use Public Transit to Get to Work?" http://overflow.solutions/demographic- data/what-percentage-of-americans-use-public-transit-to-get-to-work/ December 3, 2017

[24] Amy Forni 2017, September 5 "Four Areas Driving Autonomous Vehicle Adoption" https://www.gartner.com/smarterwithgartner/4areas-driving-autonomous-vehicle-adoption/ December 2, 2017

[25] Shaygan A., Ozdemir D., Kutgun H., Daneshi A, Adoption Criteria Evaluation of Activity Tracking Wristbands for University Students, PICMET 2017, Portland, Oregon

[26] Shaygan, A. and Testik, Ö.M., 2017. A fuzzy AHP-based methodology for project prioritization and selection. Soft Computing, pp.111. https://doi.org/10.1007/s00500-017-2851-9. 